\title{
Constraints on the range $\lambda$ of Yukawa-like modifications to the Newtonian inverse-square law of gravitation from Solar System plan- etary motions
}

\author{
Lorenzo Iorio \\ Viale Unità di Italia 68, 70125 \\ Bari(BA), Italy \\ tel. 00393286128815 \\ e-mail: lorenzo.iorio@libero.it
}

\begin{abstract}
In this paper we use the latest corrections to the Newton-Einstein secular rates of perihelia of some planets of the Solar System, phenomenologically estimated with the EPM2004 ephemerides by the Russian astronomer E.V. Pitjeva, to put severe constraints on the range parameter $\lambda$ characterizing the Yukawa-like modifications of the Newtonian inverse-square law of gravitation. It turns out that the range cannot exceed about one tenth of an Astronomical Unit. We assumed neither equivalence principle violating effects nor spatial variations of $\alpha$ and $\lambda$. This finding may have important consequences on all the modified theories of gravity involving Yukawa-type terms with range parameters much larger than the Solar System size. However, caution is advised since we, currently have at our disposal only the extra-rates of periehlia estimated by Pitjeva: if and when other groups will estimate their own corrections to the secular motion of perihelia, more robust and firm tests may be conducted.
\end{abstract}

Keywords: Modified theories of gravity; Experimental tests of gravitational theories; Celestial mechanics; Orbit determination and improvement; Ephemerides, almanacs, and calendars

PACS: 04.50.Kd; 04.80.Cc; 95.10.Ce; 95.10.Eg; 95.10.Km

\section{Introduction}

Historically, the first attempts to find deviations from the Newtonian inversesquare law of gravitation were performed to explain the anomalous secular precession of Mercury's perihelion discovered by Le Verrier (1859): 
Hall (1894) noted that he could account for Mercury's precession if the law of gravity, instead of falling off as $1 / r^{2}$, actually falls of as $1 / r^{k}$ with $k=2.00000016$. However, such an idea was not found to be very appealing, since it conflicts with basic conservation laws, e.g., Gauss's Law, unless one also postulates a correspondingly modified metric for space. Other historical attempts to modify Newton's law of gravitation to account for the Mercury's perihelion behavior yielded velocity-dependent additional terms: for a review of them see (Giné, 2007) and references therein. Such attempts practically ceased after the successful explanation of the perihelion rate of Mercury by Einstein (1915) in terms of his general theory of relativity: an exception is represented by Manev (1930) who, with a $1 / r^{2}$ correction to the Newtonian potential, was able to reproduce the anomalous apsidal precession of Mercury.

It was recently realized that deviations from the Newton's inverse-square law could provide windows into new physics (Fuji, 1991; Fischbach et al., 1992). Indeed, in the modern framework of the challenge of unifying gravity with the other three fundamental forces of Nature possible new phenomena could show up as deviations from the inverse-square law of gravitation. In general, they would occur at submillimeter length scales, but sometimes also at astronomical or even cosmological distances. For a review of the many theoretical speculations about deviations from the $1 / r^{2}$ law see (Adelberger et al., 2003).

Among various parameterizations like, e.g., power-law (Fischbach et al., 2001), a very popular, phenomenological way to account for a possible violation of the Newtonian inverse-square law takes the form of a Yukawa-like, exponentially modified Newtonian potential

$$
U=-\frac{G M}{r}\left[1+\alpha \exp \left(-\frac{r}{\lambda}\right)\right]
$$

where $G$ is the Newtonian gravitational constant, $M$ is the mass of the central body which acts as source of the gravitational field, $\alpha$ and $\lambda$ are the strength $^{1}$ and the range, respectively, of the putative new interaction. The Yukawa correction to the Newtonian potential

$$
U_{\mathrm{Y}}=-\frac{G M \alpha}{r} \exp \left(-\frac{r}{\lambda}\right)
$$

\footnotetext{
${ }^{1}$ Here we will not consider composition-dependent $\alpha$ which would induce violations of the equivalence principle; for a derivation of the potential of eq. (1) in a relativistic gravity model obeying the equivalence principle see (Zhytnikov and Nester, 1994).
} 
yields an entirely radial extra-acceleration

$$
A_{\mathrm{Y}}=-\frac{G M \alpha}{r^{2}}\left(1+\frac{r}{\lambda}\right) \exp \left(-\frac{r}{\lambda}\right) .
$$

For a review of various theoretical frameworks (braneworld models, scalartensor or scalar-tensor-vector theories of gravity, studies of topological defects) yielding a Yukawa-like, fifth force see, e.g., (Krause and Fischbach, 2001; Bertolami and Páramos, 2005; Bertolami et al., 2006; Moffat, 2006a) and references therein. Among them, there are various models of modified gravity which predict effects at astronomical scales or even larger. For example, the recent Scalar-Tensor-Vector Gravity (STVG) by Moffat (2006a) in the intentions of his proponent would be able to comprehensively and consistently account for the observed data in the Solar System, the Galaxy, clusters of galaxies and cosmological scenarios (Moffat, 2006b). Other studies on long-range, Yukawa-like modifications of gravity conducted with different techniques on astronomical/astrophysical scales can be found in (White and Kochanek, 2001; Amendola and Quercellini, 2004; Sealfon et al., 2005; Reynaud and Jaekel, 2005; Shirata et al., 2005; Sereno and Peacock, 2006).

The problem of finding experimental or observational constraints on the parameters $\alpha$ and $\lambda$, which is crucial to exclude unviable models and achieve some progress in the study on those that appear feasible, is usually tackled by looking at what happens at $\alpha$ by keeping $\lambda$ fixed, and subsequently repeating the process by sampling different spatial ranges for $\lambda$, without asking if this or that particular range for $\lambda$ is, in fact, really allowed: see, e.g. (Mikkelsen and Newman, 1977; De Rújula, 1986; Burgess and Cloutier, 1988; Fischbach and Talmadge, 1999; Krause and Fischbach, 2001; Bertolami and Nunes, 2003; Sereno and Jetzer, 2006; Iorio, 2007a).

In this paper we will test, in a purely phenomenological way, a very definite and widely used assumption in many modified theories of gravity, i.e. the hypothesis that $\lambda$ may assume values of the same order of magnitude, or larger than the typical sizes of the planetary orbits in the Solar System (Moffat, 2006b). We will show that Solar System tests are, in fact, able to tell us something important about ranges $\lambda \gg 10^{11} \mathrm{~m}$. To this aim, we will, first, derive an explicit expression of the secular, i.e. averaged over one orbital revolution, perihelion precession induced by a Yukawa-type anomalous acceleration on the orbits of the Solar System planets. Then, we will compare our formula, obtained in the small eccentricity approximation, with the latest estimated corrections of the perihelion ${ }^{2}$ rates (Pitjeva,

\footnotetext{
${ }^{2}$ The perihelia, as the other Keplerian orbital elements, are not observable quantities: ranges, range-rates, right ascensions, declinations are, in fact, measured.
} 
2005a) in order to see if results consistent with the tested hypothesis are obtained. About the methodology adopted, it is important to note that the corrections to the perihelion rates determined in (Pitjeva, 2005a) are phenomenologically estimated quantities of a global, least-square solution in which only Newtonian and Einsteinian dynamics was modeled: no exotic dynamical terms were included in the fit. Thus, in our opinion, such phenomenological corrections can genuinely be used to get information on a hypothetic, unmodeled force. It may be interesting to note that a somewhat independent test of the reliability of such a strategy can be found in (Iorio, $2007 \mathrm{~b}$ ) in which the mass of the Kuiper Belt Objects was assessed with the extra-rates of perihelia by Pitjeva (2005a) by obtaining results compatible with other estimates from different, non-dynamical techniques. If and when other groups will estimate their own corrections to perihelion rates we will use such determinations as well in order to enforce and extend our test. A complementary approach which could be followed consists in repeating the global fit of the Solar System data by modifying the dynamical force models of the data reduction softwares with the addition of the investigated non-standard acceleration term and, accordingly, including in the set of the parameters to be estimated in the least-square sense the ones connected with the Yukawa potential as well, so to look at their mutual correlations as well ${ }^{3}$ : however, such a strategy would be model-dependent and might yield just the outcomes desired by the experimentalist.

\section{The effects of a Yukawa-like fifth force on the perihelia}

To be more definite, let us suppose that a given theory, for various theoretical and/or observational reasons, makes use of a $\lambda$ quite larger than the typical spatial scales of the Solar System, e.g. because of a fit of a data set of a physical system different from it. In this case, an independent test of such an assumption is to check if a $\lambda$ with such characteristics yield, in fact, results compatible with the determined Solar System dynamics, within the associated errors. Clearly, should un-physical and/or inconsistent results be obtained, the considered model(s) and the related hypothesis would be ruled out.

Let us, now, work out the orbital effects induced by eq. (3), treated as a small perturbation of the Newtonian monopole term, on the planetary

\footnotetext{
${ }^{3}$ According to some people, this would be the only trustable approach to the problem.
} 
motions of the Solar System planets. In view of a direct comparison with the latest estimated extra-rates of the longitude of perihelion $\varpi$, we will consider the secular precession of such an element. For a radial perturbing acceleration $A_{r}$, the Gauss equation for the variation of $\varpi$ can be written as

$$
\frac{d \varpi}{d t}=-\frac{\sqrt{1-e^{2}}}{n a e} A_{r} \cos f
$$

where $a$ is the planet's semimajor axis, $e$ is the eccentricity, $n=\sqrt{G M / a^{3}}$ is the Keplerian mean motion and $f$ is the true anomaly. In order to obtain the secular rate of $\varpi$, eq. (3) must, first, be evaluated upon the unperturbed Keplerian ellipse, given by

$$
r=a(1-e \cos E)
$$

in terms of the eccentric anomaly $E$; then, it must be inserted into the right-hand-side of eq. (4) and, finally, the integral over a complete orbital revolution must be performed. The following formulas will be used

$$
\left\{\begin{array}{c}
\cos f=\frac{\cos E-e}{1-e \cos E}, \\
d t=\frac{(1-e \cos E)}{n} d E
\end{array}\right.
$$

In the calculation, which we are going to perform by quite reasonably assuming that $\alpha$ and $\lambda$ are constant and uniform over the typical spatial and temporal scales of Solar System bodies, the expression

$$
\exp \left(\frac{a e}{\lambda} \cos E\right)
$$

appears; it prevents us from obtaining a closed form of the averaged perihelion rate because the modified Bessel functions of first kind $I_{0,1}(\mathrm{ae} / \lambda)$ would appear (Burgess and Cloutier, 1988). Let us assume $\lambda \gtrsim a e$; with this choice, we can safely use

$$
\exp \left(\frac{a e}{\lambda} \cos E\right) \approx 1+\frac{a e}{\lambda} \cos E .
$$

In the small eccentricity approximation we, thus, get

$$
\dot{\varpi} \approx \frac{\alpha \sqrt{G M a}}{2 \lambda^{2}} \exp \left(-\frac{a}{\lambda}\right)
$$

up to terms of order $\mathcal{O}\left(e^{2}\right)$. Expressions analogous to eq. (9) can be found in (Burgess and Cloutier, 1988; Talmadge et al., 1988; Reynaud and Jaekel, 
2005 ), in which quantities proportional to the perihelion advance after one orbital revolution were worked out ${ }^{4}$, and in (Sereno and Jetzer, 2006) where the the perihelion secular rate was calculated up to $O\left(e^{4}\right)$.

\section{Constraining the range and the strength of a Yukawa-like fifth force with planetary perihelia}

The formula of eq. (9) is very useful because it allows us to get important information on the size of $\lambda$. Indeed, let us write down eq. (9) for a pair of planets, say $\mathrm{A}$ and $\mathrm{B}$, and take their ratio: by assuming that both $\alpha$ and $\lambda$ do not vary with distance we get

$$
\frac{\dot{\varpi}^{(\mathrm{A})}}{\dot{\varpi}^{(\mathrm{B})}}=\sqrt{\frac{a_{\mathrm{A}}}{a_{\mathrm{B}}}} \exp \left(\frac{a_{\mathrm{B}}-a_{\mathrm{A}}}{\lambda}\right) .
$$

Note that the ratio of the rates of perihelia due to a Yuakawa-like interaction is independent of $\alpha$ not only when the approximated expression of eq. (9) is used, but also when the general expression with the Bessel function (Burgess and Cloutier, 1988) is adopted. By defining

$$
\Pi \equiv \frac{\dot{\varpi}^{(\mathrm{A})}}{\dot{\varpi}^{(\mathrm{B})}},
$$

and

$$
\Theta(\lambda) \equiv \sqrt{\frac{a_{\mathrm{A}}}{a_{\mathrm{B}}}} \exp \left(\frac{a_{\mathrm{B}}-a_{\mathrm{A}}}{\lambda}\right)
$$

it is possible to construct

$$
\Upsilon(\lambda) \equiv \Pi-\Theta(\lambda)
$$

if, for a given range of values of $\left.\lambda \gtrsim a e\right|_{\mathrm{A} / \mathrm{B}}|\Upsilon|$ turns out to be incompatible with zero, within the errors, that range for $\lambda$ must be discarded. Note that our analysis is independent of $\alpha$, assumed to be nonzero, of course. The uncertainty in $\Upsilon$ can be conservatively assessed as

$$
\delta \Upsilon(\lambda) \leq \delta \Pi+\delta \Theta(\lambda)
$$

with

$$
\delta \Pi \leq|\Pi|\left[\frac{\delta \dot{\varpi}^{(\mathrm{A})}}{\left|\dot{\varpi}^{(\mathrm{A})}\right|}+\frac{\delta \dot{\varpi}^{(\mathrm{B})}}{\left|\dot{\varpi}^{(\mathrm{B})}\right|}\right],
$$

\footnotetext{
${ }^{4}$ They are $2 \pi \dot{\varpi} / n$ (Burgess and Cloutier, 1988; Talmadge et al., 1988) and $\dot{\varpi} / n$ (Reynaud and Jaekel, 2005).
} 
Table 1: Estimated semimajor axes $a$, in $\mathrm{AU}\left(1 \mathrm{AU}=1.49597870691 \times 10^{11}\right.$ m) (Pitjeva, 2005b), and phenomenologically estimated corrections to the Newtonian-Einsteinian perihelion rates, in arcseconds per century (" $\left.\mathrm{cy}^{-1}\right)$, of Mercury, the Earth and Mars (Pitjeva, 2005a). Also the associated errors are quoted: they are in $\mathrm{m}$ for $a$ (Pitjeva, 2005b) and in ${ }^{\prime \prime} \mathrm{cy}^{-1}$ for $\dot{\varpi}$ (Pitjeva, 2005a). For the semimajor axes they are the formal, statistical ones, while for the perihelia they are realistic in the sense that they were obtained from comparison of many different solutions with different sets of parameters and observations (Pitjeva, private communication 2005). The results presented in the text do not change if $\delta a$ are re-scaled by a factor 10 in order to get more realistic uncertainties.

\begin{tabular}{ccccc}
\hline Planet & $a(\mathrm{AU})$ & $\delta a(\mathrm{~m})$ & $\dot{\varpi}\left({ }^{\prime \prime} \mathrm{cy}^{-1}\right)$ & $\delta \dot{\varpi}\left({ }^{\prime \prime} \mathrm{cy}^{-1}\right)$ \\
\hline Mercury & 0.38709893 & 0.105 & -0.0036 & 0.0050 \\
Earth & 1.00000011 & 0.146 & -0.0002 & 0.0004 \\
Mars & 1.52366231 & 0.657 & 0.0001 & 0.0005 \\
\hline & $\delta \Theta(\lambda) \leq \Theta(\lambda)\left(\left|\frac{1}{2 a_{\mathrm{A}}}-\frac{1}{\lambda}\right| \delta a_{\mathrm{A}}+\left|-\frac{1}{2 a_{\mathrm{B}}}+\frac{1}{\lambda}\right| \delta a_{\mathrm{B}}\right)$.
\end{tabular}

The linear sum of the individual errors in eq. (15) accounts for the existing correlations among the estimated perihelia corrections, which reach a maximum of about 20\% for Mercury and the Earth (Pitjeva, private communication 2005). For $\mathrm{A}=$ Earth and $\mathrm{B}=$ Mercury, Table 1 and Figure 1 tell us that $\lambda \approx a_{\text {Mer }}$ is not allowed at about $1.5-\sigma$ level; for larger heliocentric distances the constraints are quite tighter, exceeding the $3-\sigma$ level. Although the inspected range for $\lambda$ ends at Mars in Figure 1, it turns out that larger values, far beyond the Solar System boundaries, are ruled out as well at about $8-\sigma$ level. Our results are unaffected by re-scaling by a factor 10 the formal errors in the semimajor axes.

After having discovered that $\lambda$ cannot exceed the semimajor axis of Mercury, let us now further constrain it. From eq. (10) it can be obtained

$$
\lambda=\frac{a_{\mathrm{B}}-a_{\mathrm{A}}}{\ln \left(\sqrt{\frac{a_{\mathrm{B}}}{a_{\mathrm{A}}}}\right)} .
$$

It turns out that the major sources of error are the estimated extra-rates of perihelia through their ratio $\Pi$, so that

$$
\delta \lambda \leq\left|\frac{a_{\mathrm{B}}-a_{\mathrm{A}}}{\Pi \ln ^{2}\left(\sqrt{\frac{a_{\mathrm{B}}}{a_{\mathrm{A}}}}\right)}\right| \delta \Pi .
$$




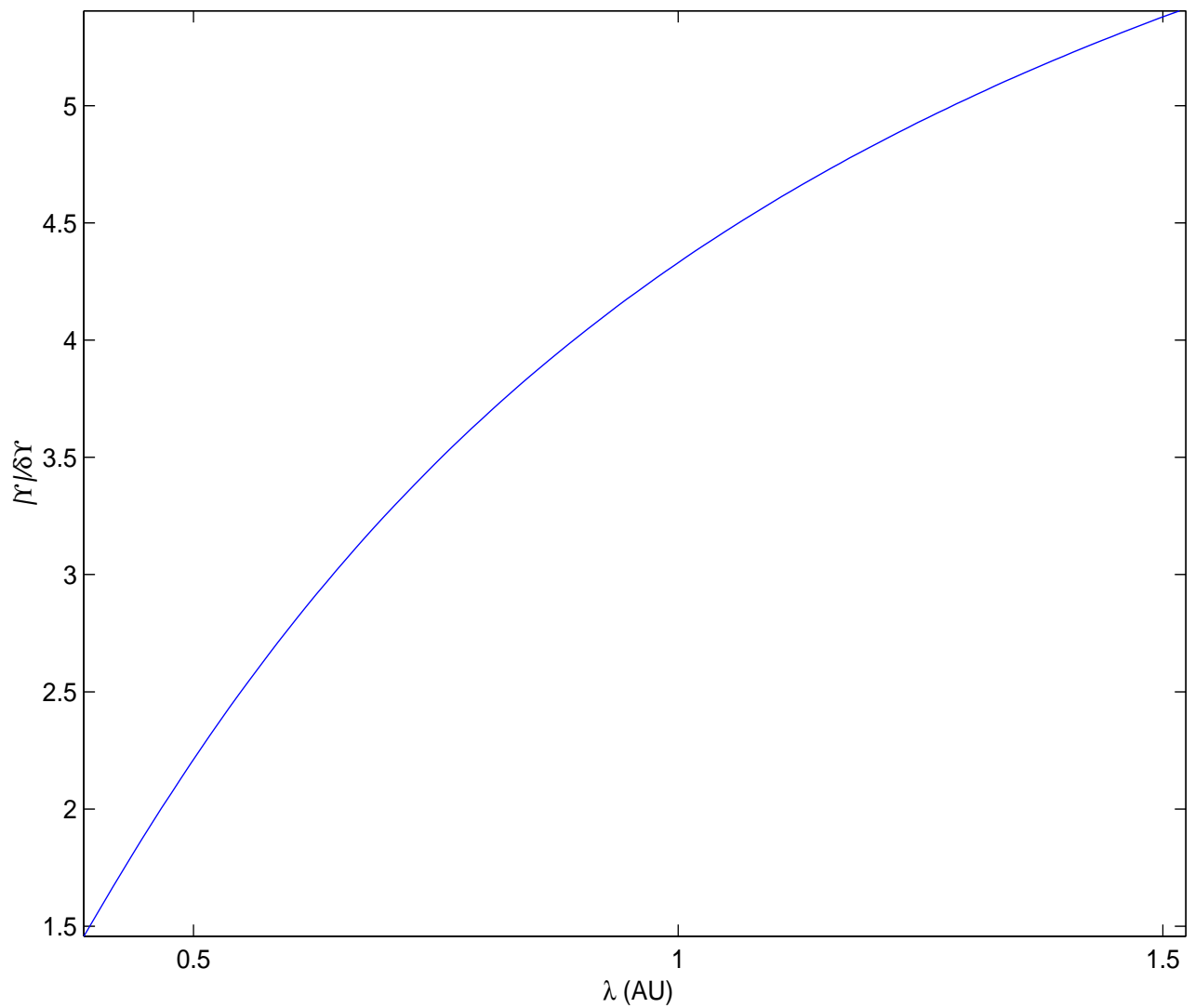

Figure 1: $|\Upsilon| / \delta \Upsilon$ from the data of the Earth and Mercury over a range $a_{\text {Mer }}<\lambda<a_{\text {Mar }}$. Values of $\lambda>a_{\text {Mar }}$ are ruled out at an even larger number of $\sigma$. 
For $\mathrm{A}=$ Earth, $\mathrm{B}=$ Mercury we have

$$
\lambda=0.182 \pm 0.183 \mathrm{AU}
$$

which is marginally compatible with zero. Note that the use of eq. (9), from which eq. (10) and eq. (17) come, can be a posteriori justified because for the Earth and Mercury the obtained value for $\lambda$ yields $a e / \lambda<1$.

The result of eq. (19) allows us to constrain $\alpha$ as well. Indeed, in the case of Mars we have

$$
\frac{a e}{\lambda}=0.78
$$

so that the approximation of eq. (8), and the formula of eq. (9) based on it, hold. Thus, from eq. (19) and the values of Table 1 for Mars we get

$$
\alpha=\frac{2 \lambda^{2} \dot{\varpi}}{\sqrt{G M a}} \exp \left(\frac{a}{\lambda}\right)=2 \times 10^{-10} .
$$

The uncertainty can be evaluated as

$$
\delta \alpha \leq \alpha\left(\frac{1}{\lambda}\left|2-\frac{a}{\lambda}\right| \delta \lambda+\frac{1}{|\dot{\varpi}|} \delta \dot{\varpi}\right)=1.3 \times 10^{-9} .
$$

Also in this case, $\alpha$ is compatible with zero. Such constrains on $\alpha$ are less tight than those obtained, e.g., in (Iorio, 2007a; Sereno and Jetzer, 2006), but the authors of such works made use of values of $\lambda$ which the present analysis has ruled out.

\section{Conclusions}

In this paper we put on the test the hypothesis that modifications of the Newtonian inverse-square law, parameterized in terms of a Yukawa-like correction, can occur over astronomical scales by using the corrections to the Newtonian-Einsteinian secular rates of the perihelia of Mercury and the Earth phenomenologically estimated, in the least-square sense, with the EPM2004 ephemerides by Pitjeva (2005a).

By taking their ratio we found that the range parameter $\lambda$ of a Yukawalike fifth force cannot exceed about 0.18 AU. The determined extra-precession of the perihelion of Mars yielded an upper bound on $\alpha$ of $10^{-9}$, which is compatible with other estimates obtained with other approaches. The values obtained for both $\lambda$ and $\alpha$ are compatible with zero; moreover, the results presented here are left unaffected by re-scaling the uncertainties in the estimated Keplerian orbital elements by a factor 10 in order to evaluate them more realistically. 
If and when corrections to the secular rates of perihelia will be estimated by other teams of astronomers, more complete and extensive tests could be performed.

Another approach which could be followed consists in introducing an adhoc Yukawa-type term in the dynamical force models of the ephemeridesgenerating routines and repeating the global fit of the whole Solar System data set by estimating, among other things, also the parameters in terms of which the new force is expressed.

\section{References}

Adelberger, E.G., Heckel, B.R., and Nelson, A.E., Tests of the Gravitational Inverse-Square Law, Ann. Rev. Nucl. Part. Sci, 53, 77-121, 2003.

Amendola, L., and Quercellini, C., Skewness as a Test of the Equivalence Principle, Phys. Rev. Lett., 92(18), 181102, 2004.

Bertolami, O., and Nunes, F.M., Ultracold neutrons, quantum effects of gravity and the weak equivalence principle, Class. Quantum Grav., 20(5), L61-L66, 2003.

Bertolami, O., and Páramos, J., Astrophysical Constraints on Scalar Field Models, Phys. Rev. D, 71(2), 023521, 2005.

Bertolami, O., Páramos, J., and Turyshev, S., General Theory of Relativity: Will it survive the next decade?, in: Dittus, H., Lämmerzahl, C., Turyshev, S. (eds.), Lasers, Clocks, and Drag-Free: Technologies for Future Exploration in Space and Tests of Gravity, pp. 27-67, (Springer, 2006).

Burgess, C.P., and Cloutier, J., Astrophysical evidence for a weak new force?, Phys. Rev. D, 38(10), 2944-2950, 1988.

De Rújula, A., On weaker forces than gravity, Phys. Lett. B, 180(3), 213$220,1986$.

Einstein, A., Erklärung der Perihelbewegung des Merkur aus der allgemeinen Relativitätstheorie, Sitzungsber. Kön. Preuss. Akad. Wiss. (Berlin), 47(2), 831-839, 1915.

Fischbach, E., Gillies, G.T., Krause, D.E., Schwan, J.G., and Talmadge, C., Non-Newtonian Gravity and New Weak Forces: an Index of Measurements and Theory, Metrologia, 29(3), 213-260, 1992. 
Fischbach, E., and Talmadge, C.L., The search for non-Newtonian gravity, (Springer-Verlag, New York 1999).

Fischbach, E., Krause, D.E., Mostepanenko, V.M., and Novello, M., New constraints on ultrashort-ranged Yukawa interactions from atomic force microscopy, Phys. Rev. D, 64(7), 075010, 2001.

Fujii, Y., The Theoretical Background of the Fifth Force, Int. J. Mod. Phys. A, 6(20), 3505-3557, 1991.

Giné, J., On the origin of the anomalous precession of Mercury's perihelion, Chaos Solitons Fractals, doi:10.1016/j.chaos.2007.02.010, 2007.

Hall, A., A suggestion in the theory of Mercury, Astron. J., 14(319), 49-51, 1894.

Iorio, L., First preliminary tests of the general relativistic gravitomagnetic field of the Sun and new constraints on a Yukawa-like fifth force from planetary data, Planet. Space Sci., 55(10), 1290-1298, 2007 a.

Iorio, L., Dynamical determination of the Kuiper Belt's mass from motions of the inner planets of the Solar System, Mon. Not. Roy. Astron. Soc., 375(4), 1311-1314, 2007b.

Krause, D.E., and Fischbach, E., Searching for Extra-Dimensions and New String-Inspired Forces in the Casimir Regime, in: Lämmerzahl, C., Everitt, C.W.F., and Hehl, F.W. (eds.), Gyros, Clocks, Interferometers...: Testing Relativistic Gravity in Space, pp. 292-309, (SpringerVerlag, Berlin, 2001).

Le Verrier, U., Lettre de M. Le Verrier à M. Faye sur la théorie de Mercure et sur le mouvement du périhélie de cette planète, Comptes Rendus de l'Académie des Sciences (Paris), 49, 379-383, 1859.

Manev, G., La gravitation et l'ènergie au zéro, Comptes Rendus de l'Académie des Sciences (Paris), 190, 1374-1377, 1930.

Mikkelsen, D.R., and Newman, M.J., Constraints on the gravitational constant at large distances, Phys. Rev. D, 16(4), 919926, 1977.

Moffat, J.W., Scalar-Tensor-Vector Gravity Theory, J. Cosmol. Astropart. Phys., 3, 4, 2006a. 
Moffat, J.W., A Modified Gravity and its Consequences for the Solar System, Astrophysics and Cosmology, Talk given at the "International Workshop From Quantum to Cosmos: Fundamental Physics in Space", 22-24 May, 2006, Warrenton, Virginia, USA.,2006, http://arxiv.org/abs/gr-qc/0608074

Pitjeva, E.V., Relativistic Effects and Solar Oblateness from Radar Observations of Planets and Spacecraft, Astron. Lett., 31(5), 340-349, 2005a.

Pitjeva, E.V., High-Precision Ephemrides of Planets-EPM and Determinations of Some Astronomical Constants. Solar Sys. Res., 39(3), 176-186, 2005b.

Reynaud, S., and Jaekel, M.-T., Testing the Newton Law at Long Distances, Int. J. Mod. Phys. A, 20(11), 2294-2303, 2005.

Sealfon, C., Verde, L., and Jimenez, R., Limits on deviations from the inverse-square law on megaparsec scales, Phys. Rev. D, 71(8), 083004, 2005 .

Sereno, M., and Peacock, J.A., Imprints of deviations from the gravitational inverse-square law on the power spectrum of mass fluctuations, Mon. Not. Roy. Astron. Soc., 371(2), 719-726, 2006.

Sereno, M., and Jetzer, Ph., Dark matter vs. modifications of the gravitational inverse-square law. Results from planetary motion in the solar system, Mon. Not. Roy. Astron. Soc., 371, 626-632, 2006.

Shirata, A., Shiromizu, T., Yoshida, N., and Suto, Y., Galaxy clustering constraints on deviations from Newtonian gravity at cosmological scales, Phys. Rev. D, 71(6), 064030, 2005.

Talmadge, C., Berthias, J.-P., Hellings, R.W., and Standish, E.M., ModelIndependent Constraints on Possible Modifications of Newtonian Gravity, Phys. Rev. Lett., 61(10), 1159-1162, 1988.

White, M., and Kochanek, C.S., Constraints on the Long-Range Properties of Gravity from Weak Gravitational Lensing, Astrophys. J., 560(2), 539$543,2001$.

Zhytnikov, V.V., and Nester, J.M., Can galactic observations be explained by a relativistic gravity theory?, Phys. Rev. Lett., 73(22), 2950-2953, 1994. 